\title{
Sex Differences in Diet and Cardio-Metabolic Diseases in the Pacific Islands ${ }^{\dagger}$
}

\author{
Briar McKenzie 1,*, Joseph A. Santos ${ }^{1}$, Claire Johnson ${ }^{1}$, Sanne A. E. Peters ${ }^{2}$, Mark Woodward 1,2,3 \\ and Jacqui Webster ${ }^{1}$ \\ 1 The George Institute for Global Health, University of New South Wales, Sydney 2042, Australia; \\ jsantos@georgeinstitute.org.au (J.A.S.); cjohnson@georgeinstitute.org.au (C.J.); \\ markw@georgeinstitute.org.au (M.W.); jwebster@georgeinstitute.org.au (J.W.) \\ 2 The George Institute for Global Health, Oxford University, Oxford OX1 2BQ, UK; \\ sanne.peters@georgeinstitute.ox.ac.uk \\ 3 Department of Epidemiology, John Hopkins University, Baltimore, MD 21218, USA \\ * correspondence: bmckenzie@georgeinstitute.org.au \\ + Presented at the 2018 Nutrition Society of New Zealand Annual Conference, Auckland, New Zealand, \\ 28-30 November 2018.
}

Published: 7 March 2019

Background: The burden of non-communicable diseases (NCDs) is increasing in low-to middleincome countries (LMIC), increasing disproportionately for women in LMIC compared to women in high income countries. Pacific Island Nations (PIs) have some of the highest rates of NCDs globally, and account for nine of the most obese nations. With the transition from a traditional to a westernised diet, nutritional intake is a key modifiable risk factor. Given the United Nations Sustainable Development Goals of good health and wellbeing and gender equality, understanding sex differences in dietary intake and related health outcomes is needed to tailor interventions.

Methods: A systematic review was conducted in January 2018. Studies included provided information on diet, disaggregated by sex in a general PI population (15-64 yrs). Electronic databases and grey literature were searched. Sex differences in dietary intake were described and differences in diet related NCD risk factors (body mass index (BMI), hypertension, dyslipidaemia and high blood glucose) assessed. Where possible, studies were combined in a meta-analysis to quantify differences.

Results: Of 1529 records found, 22 met the inclusion criteria. These studies included information on energy $(n=6)$, fat (6), carbohydrate (2), salt (2) and fruit and vegetable (13) consumption. Men had a higher energy intake than women by $264(95 \%$ CI 238, 290) kcal/day. Women consumed fruit and vegetables slightly, but significantly, more frequently than men, by $0.14(0.23,0.05)$ and $0.24(0.40$, 0.08) days/week, respectively. Women had higher BMIs than men in all 13 countries surveyed, by $2.30(2.97,1.64) \mathrm{kg} / \mathrm{m}^{2}$, overall. Conversely, systolic blood pressure was higher in men, by 6.93 (4.89, 8.97) $\mathrm{mmHg}$. Remaining risk factors showed no differences.

Conclusions: These findings illustrate sex differences in diet and important differences in BMI and systolic blood pressure in the PIs. There is a need for comprehensive data on diet and hard cardiometabolic outcomes to explore long-term ramifications.

(C) 2019 by the authors. Licensee MDPI, Basel, Switzerland. This article is an open access article distributed under the terms and conditions of the Creative Commons Attribution (CC BY) license (http://creativecommons.org/licenses/by/4.0/). 